\title{
Factors associated with rifampin resistance in staphylococcal periprosthetic joint infections (PJI): a matched case-control study
}

\author{
Y. Achermann $\cdot$ K. Eigenmann $\cdot$ B. Ledergerber $\cdot$ \\ L. Derksen $\cdot$ P. Rafeiner $\cdot$ M. Clauss $\cdot$ R. Nüesch \\ C. Zellweger $\cdot$ M. Vogt $\cdot$ W. Zimmerli
}

Received: 19 July 2012/ Accepted: 18 August 2012/Published online: 19 September 2012

(C) Springer-Verlag 2012

\begin{abstract}
Purpose Rifampin combination therapy plays an important role in the management of staphylococcal periprosthetic joint infection (PJI). However, the emergence of rifampin resistance is a feared complication. We retrospectively analysed predetermined potential risk factors in patients with rifampin-resistant staphylococcal PJI in a multicentre case-control study.

Methods Cases $(n=48)$ were defined as PJI caused by rifampin-resistant staphylococci. Rifampin-susceptible controls $(n=48)$ were matched for microorganism and type of prosthetic joint. Uni- and multivariable conditional logistic regression analyses were performed to estimate
\end{abstract}

Y. Achermann · B. Ledergerber

University and University Hospital Zurich, Zurich, Switzerland

Y. Achermann · K. Eigenmann · M. Vogt

Cantonal Hospital Zug, Baar, Switzerland

L. Derksen · W. Zimmerli ( $₫)$

Basel University Medical Clinic, Rheinstrasse 26,

4410 Liestal, Switzerland

e-mail: werner.zimmerli@unibas.ch

P. Rafeiner

Cantonal Hospital St. Gallen, St. Gallen, Switzerland

M. Clauss

Clinic of Orthopedic Surgery, Liestal, Switzerland

R. Nüesch

Clinic Hirslanden, St. Anna Lucerne, Switzerland

C. Zellweger

Clinic Hirslanden Beau-Site, Bern, Switzerland

M. Vogt

Orthopedic Clinic Schulthess, Zurich, Switzerland odds ratios (OR) with $95 \%$ confidence intervals (95\% CI).

Results Forty-eight cases (31 men; median age 67 years; age range $39-88$ years) with hip- $(n=29)$, knee$(n=13)$, elbow- $(n=4)$, shoulder- $(n=1)$ or ankle-PJI $(n=1)$ were enrolled in the study. Staphylococcus aureus and coagulase-negative staphylococci were isolated in ten and 38 episodes, respectively. Most of the cases $(n=44$, $92 \%)$ had a previous PJI, and $93 \%(n=41)$ of these had been treated with rifampin. There was an independent association of emergence of rifampin resistance with male sex (OR 3.6, $95 \%$ CI 1.2-11), $\geq 3$ previous surgical revisions (OR 4.7, $95 \%$ CI 1.6-14.2), PJI treatment with high initial bacterial load (inadequate surgical debridement, $<2$ weeks of intravenous treatment of the combination medication; OR 4.9, $95 \% \mathrm{CI}$ 1.6-15) and inadequate rifampin therapy (OR 5.4, $95 \%$ CI 1.2-25).

Conclusions Based on our results, extensive surgical debridement and adequate antibiotic therapy are needed to prevent the emergence of rifampin resistance.

Keywords Rifampin - Periprosthetic joint infection . Staphylococci - Antibiotic resistance $\cdot$ Case-control study

\section{Introduction}

During the last 20 years, rifampin has been increasingly used in implant-associated staphylococcal infections [1-9]. Microorganisms grow and persist as a biofilm on the implant surface and, consequently, such infections are difficult to eradicate. The definite cure of biofilm infections requires adequate surgical debridement and prolonged antimicrobial therapy [10]. Rifampin is active against such infections because it has a low minimal bactericidal concentration 
against Staphylococcus aureus and coagulase-negative staphylococci (CNS) in the stationary phase of growth [1114]. It has been shown to cure experimental implant-associated staphylococcal infections in animal models and has been found to be more efficacious than standard therapy in observational studies as well as in a controlled trial of patients with orthopaedic device-associated infection [1, 36, 15-19]. However, when given as monotherapy, the emergence of resistance is frequent. Resistance occurs by a single-step mutation in the DNA-dependent rRNA polymerase (alterations in the $r p o B$ gene) with a frequency of $10^{-8}$ [20-24]. Therefore, rifampin should always be used in combination with another active antibiotic. There are additional potential risk factors for the emergence of resistance. Patients with a compromised skin barrier (open wound, sinus tract or postoperative drainage) who are treated with rifampin are potentially at risk for acquiring a superinfection caused by selected rifampin-resistant skin bacteria. Furthermore, the risk for emergence of rifampin resistance is increased if the bacterial load at the start of treatment is high, i.e. as in the case of an abscess. This situation may arise if debridement surgery and/or initial treatment is not performed with a concomitant intravenous antibiotic.

The aim of this study was to analyze potential risk factors for the emergence of rifampin resistance in a retrospective case-control study of patients with staphylococcal periprosthetic joint infection (PJI). Such data would allow strategies to be developed for the prevention of rifampin resistance.

\section{Methods}

Study design and population

In this multicentre case-control study, factors associated with rifampin resistance in patients with staphylococcal PJI were analysed. Patients suffering from staphylococcal PJI between January 2001 and April 2010 were retrospectively included in the study. Data from clinical, demographic, microbiological, antimicrobial treatment and surgical intervention from primary implantation and all revisions were summarized with a standardized case report form (CRF) by an infectious diseases service physician of each clinic. One author (YA) analysed all data from the different medical centers as an independent person.

Cases were identified in the laboratory registers of all participating study centres. In two centres (Clinic Schulthess, Zurich; University Clinic of Liestal) cases were also crosschecked for completeness with information obtained from a prospective database of the Infectious Diseases Consultation Service and from the prospective Orthopaedic Prosthetic Joint Cohort database. Inclusion criteria for a case patient were (1) age $\geq 16$ years and (2) PJI during the period from January 2001 and April 2010 that was caused by $S$. aureus or CNS resistant to rifampin, as determined by microdilution or the strip test (E-Test). The exclusion criterion was an incomplete medical chart, i.e. no complete information about the preceding PJI (referring hospital, no storage of patient chart, incomplete data on antibiotic and surgical treatment). In the whole study population, one case was matched with one control for the infecting agent (S. aureus or CNS) and the affected joint (hip, knee, elbow, ankle, shoulder). Control patients with a susceptible staphylococcal PJI were all identified from the database of the Infectious Diseases Service of the Orthopaedic Center Schulthess in Zurich. This centre has a complete database excluding a selection bias of the controls.

\section{Definitions}

Prosthetic joint infection was diagnosed if one or more of the following criteria were fulfilled: (1) visible purulence of a preoperative aspirate or intraoperative periprosthetic tissue (as determined by the surgeon), (2) presence of a sinus tract communicating with the prosthesis, (3) microbial growth in a preoperative joint aspirate, intraoperative periprosthetic tissue or sonication fluid of the removed implant or (4) synovial fluid with $>1,700$ leukocytes/ $\mu 1$ or $>65 \%$ granulocytes [25-27]. These diagnostic criteria are well accepted and have been used in several previous studies of PJI [2, 28].

Inadequate rifampin treatment was defined as (1) monotherapy, (2) empiric rifampin combination with a narrow-spectrum antibiotic (e.g. clindamycin or flucloxacillin) or (3) combination therapy with an oral antibiotic with low bioavailability (e.g. oral betalactam) or an inadequate low dose.

\section{Potential risk factors for emergence of rifampin} resistance

We looked for documentation of the following potential risk factors for rifampin resistance: (1) repeated previous surgical revisions, (2) previous PJI treated with any type of antibiotics, (3) previous rifampin therapy, (4) previous inadequate rifampin treatment, (5) prolonged wound discharge ( $>14$ days) or sinus tract (6) expected high bacterial load at start of antibiotic treatment, as defined by $<2$ weeks of initial intravenous therapy and/or no surgical debridement surgery.

Statistical analysis

In this multicentre retrospective case-control study we matched one case with one control for the following two 
groups: (1) infecting agent (S. aureus or CNS) and (2) localization of PJI (hip, knee, elbow, ankle, shoulder). Associations between rifampin resistance and clinical and demographic characteristics were assessed by univariable and multivariable conditional logistic regression analyses. For statistical analysis, we used Stata software ver. 10.1 (StataCorp, College Station, TX).

Characteristics/factors studied were age at infection, sex, underlying joint disease, time to infection from last surgical revision, microbiological characteristics of infection (mono- or polymicrobial infection, methicillin resistance), previous number of revisions, previous history of PJI treated with antibiotics, previous exposure to rifampin (adequate or inadequate), high density of staphylococci at start of antibiotic treatment (no surgical debridement or initial intravenous therapy for less than 2 weeks), sinus tract or wound discharge. The multivariable models were based on factors with $P$ values of $\leq 0.1$ in the univariable analyses.

The study protocol was approved by the Institutional Review Boards of the respective institutions.

\section{Results}

Baseline characteristics of the 48 cases with rifampinresistant staphylococcal PJI

Baseline characteristics of the 48 patients with rifampinresistant staphylococcal PJI are summarized in Table 1. The median age of these patients was 67 years (range 39-88 years), and 31 (65\%) were male. Infected devices were hip- $(n=29,60 \%)$ knee- $(n=13,27 \%)$, elbow$(n=4,8 \%)$ and ankle- and shoulder-prostheses (one each, $2 \%$ ). The indications for joint replacement were osteoarthritis (34 patients, $71 \%$ ), rheumatic disorders (4, $8 \%$ ) and trauma $(10,21 \%)$. Ten cases of rifampin-resistant PJI were caused by $S$. aureus and 38 by CNS. Of the staphylococcal isolates resistant to rifampin, 31 (65\%) of the CNS isolates, but none of the $S$. aureus isolates, were methicillin-resistant. Most of the patients had previous revision surgery, such as debridement or exchange of the prosthesis due to any reason (median revision surgery 3.1, range $0-25)$. The median time to infection after the last surgical procedure was 5.1 months.

Almost half of the patients $(46 \%)$ had a previous sinus tract or a prolonged wound discharge of $>14$ days due to PJI. Most of the patients with a previous PJI were treated with antibiotics $(n=44,92 \%)$, including rifampin $(n=41,85 \%)$. In 25 of 41 patients $(61 \%)$, previous rifampin therapy was correctly given. However, four patients had either monotherapy $(n=1)$ or a combination with an inactive drug $(n=3)$. Seven patients were previously treated with a rifampin combination for a culture-negative PJI, and seven other patients had a combination either with an underdosed drug $(n=3)$ or an oral betalactam $(n=4)$. In 34 patients $(71 \%)$, intravenous antimicrobial treatment was either shorter than 2 weeks $(n=12)$ and or the patients did not receive surgical debridement $(n=22)$ at the start of therapy.

Demographic and clinical variables affecting the risk of emergence of rifampin resistance according to univariable and multivariable logistic regression analyses

Table 2 shows the results of univariable and multivariable logistic regression analyses. In the univariable model, male sex and multiple $(\geq 3)$ previous surgical revisions were significant risk factors, as well as previous PJI with a sinus tract or prolonged wound discharge ( $>14$ days) and any previous antibiotic treatment, especially with rifampin. Previous rifampin treatment is a risk factor for the emergence of rifampin resistance in general [odds ratio (OR) 7.8], and even more so if given in an inadequate manner (OR 10.2). Finally, a short initial intravenous treatment $(<2$ weeks $)$ and lack of surgical debridement were also significant risk factors.

Analysis using a multivariable model (Table 2) revealed the following independent risk factors: (1) male sex (OR 3.6), (2) $\geq 3$ surgical revisions (OR 4.7), (3) previous PJI treated with rifampin in inadequate (OR 5.4) manner and (4) presumably high bacterial load at start of the antibiotic treatment (OR 4.9).

\section{Discussion}

In this retrospective case-control study, we found an independent association between the emergence of rifampin resistance and male sex, $\geq 3$ previous surgical revisions, rifampin treatment without surgical debridement or $<2$ weeks of initial intravenous antibiotic therapy with an concomitant drug, as well as inadequate rifampin therapy as defined in the "Methods".

With increasing rifampin resistance, fewer patients with PJI would be able to benefit from orthopaedic implant retention. In most countries, spontaneous resistance of staphylococci to rifampin is rare. In Spain, in a survey carried out between 1999 and 2008, 0.26\% of the methicillin-sensitive Staphylococcus aureus (MSSA) and $3.26 \%$ of the methicillin-resistant $S$. aureus (MRSA) isolates were resistant to rifampin [29]. However, if rifampin is inadequately used, resistance may emerge due to a single-step point mutation [20, 22-24, 29]; thus, there is always the potential for rifampin resistance to emerge rapidly [12]. 
Table 1 Baseline characteristics of 96 patients at time of periprosthetic joint infection with rifampin-resistant staphylocci

\begin{tabular}{|c|c|c|c|}
\hline Characteristics & Cases $(n=48)$ & Controls $(n=48)$ & $P$ value $^{\mathrm{a}}$ \\
\hline \multicolumn{4}{|l|}{ Current PJI } \\
\hline Age at hospital admission, median years (range) & $67(39-88)$ & $67(39-85)$ & $0.39^{\mathrm{e}}$ \\
\hline Male gender & $31(65 \%)$ & $17(35 \%)$ & 0.004 \\
\hline Localization of joint prosthesis & & & Matching \\
\hline Hip & $29(60.4 \%)$ & $29(60.4 \%)$ & \\
\hline Knee & $13(27.1 \%)$ & $13(27.1 \%)$ & \\
\hline Shoulder & $1(2.1 \%)$ & $1(2.1 \%)$ & \\
\hline Elbow & $4(8.3 \%)$ & $4(8.3 \%)$ & \\
\hline Ankle & $1(2.1 \%)$ & $1(2.1 \%)$ & \\
\hline Underlying joint disorder & & & $0.074^{\mathrm{f}}$ \\
\hline Primary osteoarthritis & $34(70.8 \%)$ & $37(77.1 \%)$ & \\
\hline Rheumatic osteoarthritis & $4(8.3 \%)$ & $8(16.7 \%)$ & \\
\hline Posttraumatic osteoarthritis & $10(20.8 \%)$ & $3(6.3 \%)$ & \\
\hline \multicolumn{4}{|l|}{ Microorganism } \\
\hline S. aureus & $10(20.8 \%)$ & $10(20.8 \%)$ & Matching \\
\hline Coagulase-negative staphylococci & $38(79.2 \%)$ & $38(79.2 \%$ & Matching \\
\hline Polymicrobial infections & $3(6.3 \%)$ & $6(12.5 \%)$ & $0.49^{\mathrm{f}}$ \\
\hline \multicolumn{4}{|l|}{ Microbial resistance } \\
\hline Rifampin resistance & $48(100 \%)$ & $0(0 \%)$ & $<0.001^{\mathrm{f}}$ \\
\hline Methicillin resistance & $31(64.6 \%)$ & $28(58.3 \%)$ & 0.53 \\
\hline Time from last surgical revision to infection (months) & 5.1 & 5.7 & $0.18^{\mathrm{e}}$ \\
\hline Previous surgical revisions, median (range) & $3(0-11)$ & $1(0-25)$ & $0.002^{\mathrm{e}}$ \\
\hline$\geq 3$ revisions & 32 & 13 & \\
\hline Previous PJI & $44(91.7 \%)$ & $30(62.5 \%)$ & $0.001^{\mathrm{f}}$ \\
\hline \multicolumn{4}{|l|}{ Clinical signs } \\
\hline Sinus tract, wound discharge & $22(45.8 \%)$ & $12(25 \%)$ & $0.054^{\mathrm{f}}$ \\
\hline Sinus tract and/or & 14 & 7 & \\
\hline Wound discharge ( $>14$ days) & 13 & 6 & \\
\hline \multicolumn{4}{|l|}{ Treatment } \\
\hline Treated with any antibiotics & $44(91.7 \%)$ & $30(62.5 \%)$ & $0.001^{\mathrm{f}}$ \\
\hline Treated with rifampin & $41(85.4 \%)$ & $20(41.7 \%)$ & $<0.001^{\mathrm{f}}$ \\
\hline Rifampin always adequate ${ }^{\mathrm{b}}$ & 25 & 15 & \\
\hline Rifampin inadequate & 16 & 5 & \\
\hline Monotherapy and/or & 4 & 3 & \\
\hline Empiric therapy ${ }^{\mathrm{c}}$ and/or & 6 & 1 & \\
\hline Other reasons ${ }^{\mathrm{d}}$ & 7 & 1 & \\
\hline Treatment with high bacterial load & $34(70.8 \%)$ & $13(27.1 \%)$ & $<0.001^{\mathrm{f}}$ \\
\hline$<2$ weeks iv antimicrobial treatment & 12 & 4 & \\
\hline No surgical debridement & 7 & 1 & \\
\hline No iv and no surgical debridement & 15 & 8 & \\
\hline
\end{tabular}

Data are presented as the number (of patients) with the percentage in parenthesis, except where specified otherwise iv Intravenous

${ }^{a} P$ values were calculated by $\chi^{2}$ except when otherwise specified

b Adequate treatment according to published treatment algorithm [2]

${ }^{c}$ Rifampin- companion drug with a narrow spectrum against a microorganism with unknown susceptibility

${ }^{d}$ Rifampin-companion drug with low bioavailability or underdosed

e By Mann-Whitney test

f By Fisher's exact test 
Table 2 Univariable and multivariable conditional logistic regression analyses of the 96 patients (cases $N=48$ and controls $N=48$ )

\begin{tabular}{|c|c|c|c|c|}
\hline \multirow[t]{2}{*}{ Variable } & \multicolumn{2}{|c|}{ Univariable analyses } & \multicolumn{2}{|c|}{ Multivariable analyses } \\
\hline & OR $(95 \% \mathrm{CI})$ & $P$ value & OR $(95 \% \mathrm{CI})$ & $P$ value \\
\hline \multicolumn{5}{|l|}{ Current PJI } \\
\hline \multicolumn{5}{|l|}{ Age at hospital admission } \\
\hline$<60$ years & 1 (reference) & & & \\
\hline $60-75$ years & $1.44(0.55-3.8)$ & 0.46 & & \\
\hline 75 years & $0.51(0.17-1.6)$ & 0.24 & & \\
\hline Male gender & $3.4(1.5-8.1)$ & 0.005 & $3.6(1.2-11)$ & 0.023 \\
\hline \multicolumn{5}{|l|}{ Previous revisions } \\
\hline$<3$ revisions & 1 (reference) & & & \\
\hline$\geq 3$ revisions & $5.0(2.1-12)$ & $<0.001$ & $4.7(1.6-14)$ & 0.006 \\
\hline \multicolumn{5}{|l|}{ Clinical signs of previous PJI } \\
\hline Sinus tract, wound discharge & $2.5(1.1-6.1)$ & 0.035 & $2.0(0.63-6.1)$ & 0.25 \\
\hline \multicolumn{5}{|l|}{ Treatment of previous PJI } \\
\hline Treated with antibiotics & $6.5(2.0-21)$ & 0.002 & & \\
\hline Treated with rifampin & $7.8(2.9-21)$ & $<0.001$ & & \\
\hline Rifampin always adequate $^{\mathrm{a}}$ & $6.6(2.2-19.4)$ & 0.001 & $3.2(0.81-13)$ & 0.096 \\
\hline Rifampin inadequate & $10.2(2.9-36)$ & $<0.001$ & $5.4(1.2-25)$ & 0.029 \\
\hline Rifampin monotherapy & $2.9(0.48-18)$ & 0.25 & & \\
\hline Empiric therapy ${ }^{\mathrm{b}}$ & $20(2.1-185)$ & 0.009 & & \\
\hline Other reasons ${ }^{c}$ & $21(2.3-195)$ & 0.007 & & \\
\hline Treatment with high bacterial load & $5.5(2.4-13)$ & $<0.001$ & $4.9(1.6-15)$ & 0.005 \\
\hline$<2$ weeks iv treatment & $3.5(1.5-8.1)$ & 0.004 & & \\
\hline No surgical debridement & $3.5(1.4-8.8)$ & 0.006 & & \\
\hline
\end{tabular}

CI confidence interval, $O R$ odds ratio

a Adequate treatment according to published treatment algorithm [2]

${ }^{b}$ Rifampin- companion drug with a narrow spectrum against a microorganism with unknown susceptibility

${ }^{c}$ Rifampin-companion drug with low bioavailability or underdosed

${ }^{\text {d }} P$ value was calculated by Mann-Whitney test

In an experimental foreignbody infection rat model, after a 7-day-treatment with high-dose rifampin, rifampin-resistant strains appeared between day 8 and 11 at a rate of up to $60 \%$ under selective pressure [30]. Thus, there is no doubt that with increasing use, the fraction of rifampin-resistant staphylococci will rise [31]. Indeed, in a study involving patients colonized with $S$. aureus and treated for tuberculosis, rifampin resistance emerged in five of $58(8.6 \%)$ patients [21]. Interestingly, subinhibitory concentrations of ciprofloxacin induce higher frequencies of rifampin-resistant mutants under favourable in vitro conditions [32, 33]. It is therefore important to avoid clinical situations leading to the emergence or selection of rifampin-resistant staphylococci. Therefore, in our study, we evaluated risk factors for rifampin resistance in order to develop strategies for the prevention of rifampin resistance.

Our study showed that rifampin exposure is a risk factor for the emergence of resistance, if treated inadequately. The choice of the best combination drug with rifampin is crucial for the prevention of resistance. John et al. [12] showed in an animal study that the combination of vancomycin plus rifampin does not completely prevent the emergence of rifampin resistance in the case of infection by high-density MRSA. In contrast, levofloxacin or high-dose daptomycin combined with rifampin completely prevents the emergence of rifampin resistance. Sub-inhibitory concentrations of fluoroquinolones promote the emergence of rifampin resistance and, therefore, high doses should be used in combination with rifampin [12]. In addition, the results of the susceptibility testing should be known before treatment is initiated with rifampin. A delay in initiating rifampin therapy for several days does not lower the cure rate in patients with PJI treated with debridement and retention (unpublished cohort data). During the last two decades, the fraction of fluoroquinolone-resistant staphylococci has been steadily increasing; consequently, fluoroquinolone susceptibility of the infecting strain should be confirmed. In our study, six patients with rifampin-resistant staphylococcal PJI had been previously treated with a rifampin combination, with either flucloxacillin or 
clindamycin or ciprofloxacin, without prior knowledge of the infecting agent. In three other cases, the initial empiric rifampin combination with flucloxacillin or clindamycin was given for a few days before unexpected resistance to these drugs was detected. The statistical analysis showed a lower odds ratio with rifampin monotherapy than with the empiric rifampin combination treatment in culture-negative PJI. This result can be presumably be explained as being due to a longer exposure to monotherapy in comparison to when an empiric rifampin combination is used in culturenegative PJI (52.5 vs. 6.5 days). Of the 48 cases (15\%) with a rifampin-resistant staphylococcal PJI, seven had no prior exposure to rifampin. To our knowledge, no in vitro or in vivo studies have been carried out on the incidence and terms and conditions of primary resistance in staphylococcal infections. In our study, all but one of the seven infections with a primary rifampin resistance were caused by $S$. epidermidis, a pathogen that mostly presents with subclinical signs and symptoms that lead to a delay in diagnosis and probably also to an increased chance of spontaneous mutations in the $r p o B$ gene. The second highest association with the emergence of rifampin resistance in our study was a presumed high bacterial density at the start of rifampin therapy. A high bacterial load was assumed in the case of missing or inadequate initial surgical debridement (arthroscopy instead of open debridement). In addition, patients treated for $<2$ weeks with an intravenous antibiotic as combination partner after the diagnosis of PJI were also assumed to be at risk because of the high frequency of single-step mutation $\left(1: 10^{8}\right)$. In an abscess, the density of staphylococci is at least $10^{8} \mathrm{CFU} /$ $\mathrm{ml}$; it is therefore reasonable to assume that treating PJI without debridement surgery or without adequate initial concomitant high-dose intravenous therapy increases the risk for emergence of rifampin resistance. Due to the limited number of patients, we could not separately analyse whether surgical debridement of infected tissue or highdose intravenous antibiotics alone are crucial for prevention of rifampin resistance.

The rationale for multiple previous revisions ( $\geq 3$ times) being an independent risk factor in our study is not clear. It is conceivable that the risk for inoculating rifampin-resistant staphylococci from the skin to the prosthesis increases with each surgical intervention. The increased risk of males for the emergence of rifampin resistance has no obvious explanation. We found no correlation with more revisions and higher age. However, this observation seems not to be an artifact, since the male predominance was observed in both centre with the highest number of cases.

This is the first study to analyse risk factors for rifampin resistance. The strengths of this study are (1) the routine follow-up visits in all patients with PJI in all study centres, (2) the prospective data from two centres following their patients in cohort studies and (3) the standardized surgical procedures according to an algorithm in most cases. The study has three limitations. First, it is not a controlled, but an observational study, and all controls were from one single centre. However, this one centre prospectively follows all patients in a cohort study. Therefore, the risk for a selection bias was lower than the alternative, i.e. to choose controls from each center. A third limitation is the lack of MRSA, which precludes a species-specific analysis. Despite these limitations, this study provides data which should allow the emergence of rifampin-resistance to be minimized in the future.

In conclusion, the indication for rifampin combination therapy needs to be carefully evaluated. In patients with PJI, rifampin should only be used if indicated according to published evidence $[1-5,12,14,17-20,33]$. Previous rifampin exposure predisposes to the emergence of rifampin resistance, if treated inadequately. Our data suggest that extensive surgical debridement and adequate antibiotic therapy are both crucial for preventing the emergence of rifampin resistance.

Acknowledgements We acknowledge the teams of the Division of Infectious Diseases and the Service of Orthopedic Surgery of the CHUV/Lausanne Switzerland for providing data of 2 patients in this study. This work was supported by an unrestricted grant of the HansPaul Wälchli Foundation for Research (Lugano, Switzerland).

Conflict of interest None.

\section{References}

1. Drancourt M, Stein A, Argenson JN, Roiron R, Groulier P, Raoult D. Oral treatment of Staphylococcus spp. infected orthopaedic implants with fusidic acid or ofloxacin in combination with rifampicin. J Antimicrob Chemother. 1997;39:235-40.

2. Zimmerli W, Trampuz A, Ochsner PE. Prosthetic-joint infections. N Engl J Med. 2004;351:1645-54.

3. El Helou OC, Berbari EF, Lahr BD, et al. Efficacy and safety of rifampin containing regimen for staphylococcal prosthetic joint infections treated with debridement and retention. Eur J Clin Microbiol Infect Dis. 2010;29:961-7.

4. Barberan J, Aguilar L, Gimenez MJ, Carroquino G, Granizo JJ, Prieto J. Levofloxacin plus rifampicin conservative treatment of 25 early staphylococcal infections of osteosynthetic devices for rigid internal fixation. Int J Antimicrob Agents. 2008;32:154-7.

5. Berdal JE, Skramm I, Mowinckel P, Gulbrandsen P, Bjornholt JV. Use of rifampicin and ciprofloxacin combination therapy after surgical debridement in the treatment of early manifestation prosthetic joint infections. Clin Microbiol Infect. 2005;11: $843-5$.

6. Garrigos C, Murillo O, Euba G, et al. Efficacy of tigecycline alone and with rifampin in foreign-body infection by methicillinresistant Staphylococcus aureus. J Infect. 2011;63:229-35.

7. Senneville E, Lombart A, Beltrand E, et al. Outcome of diabetic foot osteomyelitis treated nonsurgically: a retrospective cohort study. Diabetes Care. 2008;31:637-42. 
8. Zimmerli W, Ochsner PE. Management of infection associated with prosthetic joints. Infection. 2003;31:99-108.

9. Mallet M, Loiez C, Melliez H, Yazdanpanah Y, Senneville E, Lemaire X. Staphylococcus simulans as an authentic pathogenic agent of osteoarticular infections. Infection. 2011;39:473-6.

10. Zimmerli W, Moser C. Pathogenesis and treatment concepts of orthopaedic biofilm infections. FEMS Immunol Med Microbiol. 2012;65:158-68.

11. Baldoni D, Hermann H, Frei R, Trampuz A, Steinhuber A. Performance of microcalorimetry for early detection of methicillin resistance in clinical isolates of Staphylococcus aureus. J Clin Microbiol. 2009;47:774-6.

12. John AK, Baldoni D, Haschke M, et al. Efficacy of daptomycin in implant-associated infection due to methicillin-resistant Staphylococcus aureus: importance of combination with rifampin. Antimicrob Agents Chemother. 2009;53:2719-24.

13. Trampuz A, Murphy CK, Rothstein DM, Widmer AF, Landmann R, Zimmerli W. Efficacy of a novel rifamycin derivative, ABI0043, against Staphylococcus aureus in an experimental model of foreign-body infection. Antimicrob Agents Chemother. 2007;51:2540-5.

14. Baldoni D, Haschke M, Rajacic Z, Zimmerli W, Trampuz A. Linezolid alone or combined with rifampin against methicillinresistant Staphylococcus aureus in experimental foreign-body infection. Antimicrob Agents Chemother. 2009;53:1142-8.

15. Saleh-Mghir A, Muller-Serieys C, Dinh A, Massias L, Cremieux AC. Adjunctive rifampin is crucial to optimizing daptomycin efficacy against rabbit prosthetic joint infection due to methicillin-resistant Staphylococcus aureus. Antimicrob Agents Chemother. 2011;55:4589-93.

16. Senneville E, Joulie D, Legout L, et al. Outcome and predictors of treatment failure in total hip/knee prosthetic joint infections due to Staphylococcus aureus. Clin Infect Dis. 2011;53:334-40.

17. Widmer AF, Gaechter A, Ochsner PE, Zimmerli W. Antimicrobial treatment of orthopedic implant-related infections with rifampin combinations. Clin Infect Dis. 1992;14:1251-3.

18. Stengel D, Bauwens K, Sehouli J, Ekkernkamp A, Porzsolt F. Systematic review and meta-analysis of antibiotic therapy for bone and joint infections. Lancet Infect Dis. 2001;1:175-88.

19. Zimmerli W, Widmer AF, Blatter M, Frei R, Ochsner PE. Role of rifampin for treatment of orthopedic implant-related staphylococcal infections: a randomized controlled trial. Foreign-Body Infection (FBI) Study Group. JAMA. 1998;20(279):1537-41.

20. Wehrli W. Rifampin: mechanisms of action and resistance. Rev Infect Dis. 1983;5[Suppl 3]:S407-11.

21. Liu Y, Cui J, Wang R, Wang X, Drlica K, Zhao X. Selection of rifampicin-resistant Staphylococcus aureus during tuberculosis therapy: concurrent bacterial eradication and acquisition of resistance. J Antimicrob Chemother. 2005;56:1172-5.

22. Sande MA, Mandell GL. Effect of rifampin on nasal carriage of Staphylococcus aureus. Antimicrob Agents Chemother. 1975;7:294-7.

23. Dunne WM Jr, Mason EO Jr, Kaplan SL. Diffusion of rifampin and vancomycin through a Staphylococcus epidermidis biofilm. Antimicrob Agents Chemother. 1993;37:2522-6.

24. Zavasky DM, Sande MA. Reconsideration of rifampin: a unique drug for a unique infection. JAMA. 1998;279:1575-7.

25. Trampuz A, Hanssen AD, Osmon DR, Mandrekar J, Steckelberg JM, Patel R. Synovial fluid leukocyte count and differential for the diagnosis of prosthetic knee infection. Am $\mathrm{J}$ Med. 2004;117:556-62.

26. Parvizi J, Jacovides C, Zmistowski B, Jung KA. Definition of periprosthetic joint infection: is there a consensus? Clin Orthop Relat Res. 2011;469:3022-30.

27. Ghanem E, Parvizi J, Burnett RS, et al. Cell count and differential of aspirated fluid in the diagnosis of infection at the site of total knee arthroplasty. J Bone Joint Surg Am. 2008;90:1637-43.

28. Trampuz A, Osmon DR, Hanssen AD, Steckelberg JM, Patel R. Molecular and antibiofilm approaches to prosthetic joint infection. Clin Orthop Relat Res. 2003;414:69-88.

29. Villar M, Marimon JM, Garcia-Arenzana JM, de la Campa AG, Ferrandiz MJ, Perez-Trallero E. Epidemiological and molecular aspects of rifampicin-resistant Staphylococcus aureus isolated from wounds, blood and respiratory samples. J Antimicrob Chemother. 2011;66:997-1000.

30. Murillo O, Garrigos C, Pachon ME, et al. Efficacy of high doses of daptomycin versus alternative therapies against experimental foreign-body infection by methicillin-resistant Staphylococcus aureus. Antimicrob Agents Chemother. 2009;53:4252-7.

31. Hellmark B, Soderquist B, Unemo M. Simultaneous species identification and detection of rifampicin resistance in staphylococci by sequencing of the rpoB gene. Eur J Clin Microbiol Infect Dis. 2009;28:183-90.

32. Tan CK, Lai CC, Liao CH, Lin SH, Huang YT, Hsueh PR. Increased rifampicin resistance in blood isolates of methicillinresistant Staphylococcus aureus (MRSA) amongst patients exposed to rifampicin-containing antituberculous treatment. Int $\mathbf{J}$ Antimicrob Agents. 2011;37:550-3.

33. Didier JP, Villet R, Huggler E, et al. Impact of ciprofloxacin exposure on Staphylococcus aureus genomic alterations linked with emergence of rifampin resistance. Antimicrob Agents Chemother. 2011;55:1946-52. 